\title{
Hepatocellular Carcinoma with Vascular Invasion and Cardiac Metastasis Causing Progressive Liver and Renal Failure: A Case Report and Literature Review
}

\author{
Yaser Khalid ${ }^{1 *}$, Neethi Dasu ${ }^{1}$, Troy Randle ${ }^{2}$, Debashis Reja ${ }^{3}$, Adrian Pearson ${ }^{2}$, Kirti Dasu ${ }^{4}$, Kailash Lal ${ }^{5}$, Richard Walters ${ }^{5}$ \\ and Nishant Parikh ${ }^{1}$
}

${ }^{1}$ Division of Internal Medicine, Rowan University School of

Medicine at Jefferson Health System, Stratford, New Jersey

${ }^{2}$ Division of Cardiovascular Medicine, South Jersey Heart Group, Virtua Lourdes Health System, Camden, New Jersey

${ }^{3}$ Division of Internal Medicine, Rutgers Robert Wood Johnson Medical School, New Brunswick, New Jersey

${ }^{4}$ Division of Biology, Syracuse University, Syracuse, New York

\author{
Corresponding author \\ Yaser Khalid, Division of Internal Medicine, Rowan University School of \\ Medicine at Jefferson Health System, Stratford, New Jersey
}

Submitted: 16 Apr 2020; Accepted: 21 Apr 2020; Published: 05 May 2020

${ }^{5}$ Division of Gastroenterology, Rowan University School of Medicine at Jefferson Health System, Stratford, New Jersey

\begin{abstract}
Hepatocellular carcinoma (HCC) is the most common primary tumor of the liver and eighth-most common cancer. The most frequent sites of metastasis are the lungs, the bones, the lymphatics, and the brain. An isolated metastasis of a HCC to the right atrium and inferior vena cava (IVC) is extremely rare. Here we discuss a case of a 61-year-old female who presented with two weeks of generalized weakness and was found to have advanced stage HCC with metastasis to the IVC and right atrium of her heart. We recommend that echocardiography should be routinely performed for all patients with advanced HCC because if metastasis to the right atrium is not detected early enough, the clinical course will be complicated by many unexpected cardiovascular complications that can be fatal. Currently the prognosis for this disease is very poor so further investigation into surgical and medical treatments needs to occur.
\end{abstract}

\section{Introduction}

$\mathrm{HCC}$ is the eighth most common cancer overall, ranking fifth among males and seventh among females [1]. It is the most common primary tumor of the liver [1]. Additionally, liver cancer is considered to be the fourth leading cause of cancer death in the entire nation with more than half a million deaths per year [2]. Around $80-90 \%$ of $\mathrm{HCC}$ occurs secondary to cirrhosis with the following causes: HBV is the most common cause worldwide and accounts for $54 \%$ cases, $\mathrm{HCV}$ is the cause of $24 \%$ of cases, and alcohol is the third most common cause. Non-alcoholic fatty liver disease (NAFLD) is the most common cause in the United States [1]. The remaining cases of HCC can develop without cirrhosis with the following causes: genetic and autoimmune conditions (such as hemochromatosis, alpha-1 antitrypsin deficiency and acute intermittent porphyria). Rarely HCC is cryptogenic likely due to undiagnosed NASH [1]. The most common sites of HCC metastasis are the lungs, bones, and lymph nodes. Cardiac metastasis from HCC is extremely rare [1]. Here we discuss a rare case of a 61-year-old female with past medical history of multiple sclerosis who presented with 2 weeks of generalized weakness. She had no known history of liver disease but was found to have advanced stage $\mathrm{HCC}$ with metastasis to the inferior vena cava (IVC) and right atrium.
Cardiac tumors in isolation are also extremely rare. Cardiac tumors are classified into primary tumors that arise from the heart or into secondary, metastatic tumors that invade the heart from other organs. The prevalence of primary cardiac tumors is $0.001-0.03 \%$ [4]. Seventy-five percent of primary cardiac tumors are benign, with myxomas representing approximately $50 \%$ of cases. Around a fourth of all cardiac tumors are malignant, with sarcomas being the most frequent kind. Secondary tumors are about 20 - to 40 -fold more common than primary tumors, and $15 \%$ of patients suffering from any form of cancer have metastases to the heart [4].

Current guidelines recommend using ultrasound and alphafetoprotein (AFP) levels as screening tests for HCC in high risk populations, which includes patients with cirrhosis and hepatitis $\mathrm{B}$ carriers. Treatment of HCC depends on the staging of the cancer and ranges from surgical resection to systemic chemotherapy with radiofrequency ablation. The results of autopsy studies indicate a $2.7-4.1 \%$ incidence of atrial metastases of HCC [3]. A correct diagnosis is important in the clinical setting since cardiac metastases are able to induce sudden cardiac arrest [3]. 


\section{Case Presentation}

A 61-year-old female with past medical history of multiple sclerosis presented with 2 weeks of generalized weakness with intermittent diarrhea. Her home medications included: baclofen, dalfampridine, glatiramer, glipizide, liraglutide, lisinopril-hydrochlorothiazide, metformin, pregabalin, and venlafaxine. On physical exam, she had bilateral lower extremity edema with right greater than left as well as erythema of right lower extremity. Laboratory data revealed blood urea nitrogen (BUN) $52 \mathrm{mg} / \mathrm{dL}$, creatinine $2.15 \mathrm{mg} / \mathrm{dL}$, total bilirubin $1.2 \mathrm{mg} / \mathrm{dL}$, direct bilirubin $0.4 \mathrm{mg} / \mathrm{dL}$, alkaline phosphatase $232 \mathrm{U} / \mathrm{L}$, ALT $65 \mathrm{U} / \mathrm{L}, \mathrm{AST} 144 \mathrm{U} / \mathrm{L}, \mathrm{GGTP} 605 \mathrm{U} / \mathrm{L}$, and lactate 2.8 $\mathrm{mg} / \mathrm{dL}$. Her transaminitis pattern was consistent with a cholestatic pattern with $\mathrm{R}$ factor of 1.1. Computed tomography of the abdomen and pelvis (CT) imaging revealed abdominal lymphadenopathy with nodularity of liver with an approximately $3-\mathrm{cm}$ mass in the right hepatic lobe:
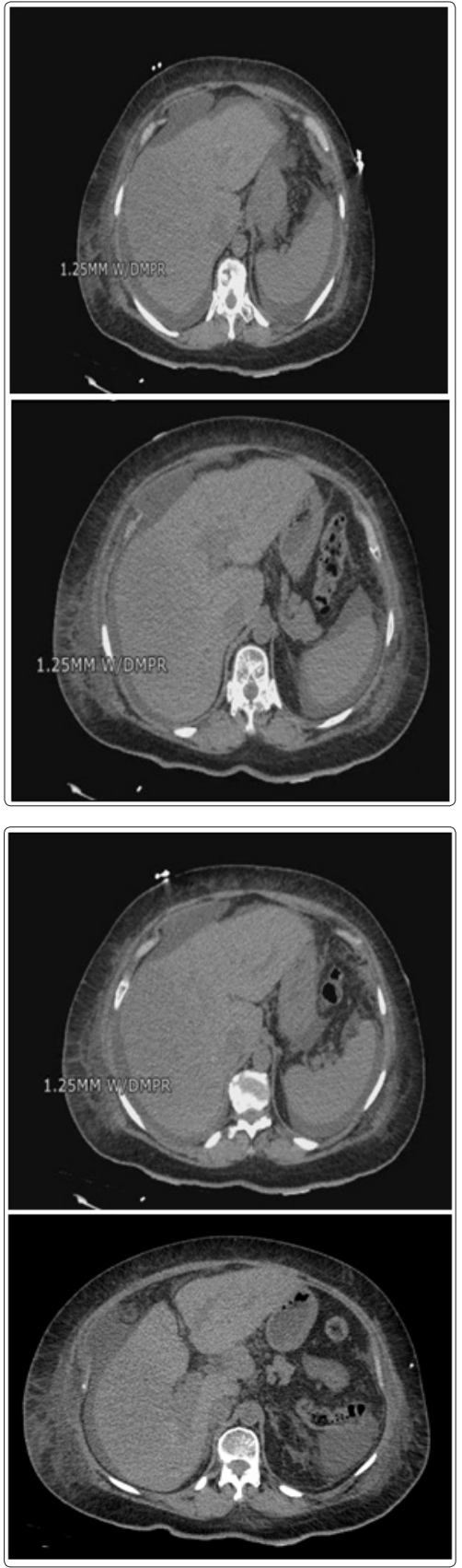

Then, the patient underwent a diagnostic paracentesis which showed a serum ascites albumin gradient (SAAG) of 1.9 and a fluid protein of $2.7 \mathrm{~g} / \mathrm{dL}$. Following the paracentesis, her mentation worsened, and she progressed to kidney and liver failure. The patient was empirically started on lactulose for suspected hepatic encephalopathy which improved her overall mentation. The patient's renal function still continued to deteriorate throughout the course of hospital stay, despite treatment with IV fluids and albumin. The patient's transaminase pattern now showed a mixed pattern of injury of both cholestatic and hepatic with an $\mathrm{R}$ factor 4 . Treatment with IV fluids for acute renal failure worsened her diffuse edema and anasarca, so the patient underwent a therapeutic paracentesis with removal of 4 liters of peritoneal fluid. Afterwards, her transaminases showed a primary hepatocellular injury pattern with $\mathrm{R}$ factor 14 . With alpha fetoprotein (AFP) levels of 1700, the diagnosis of HCC was confirmed as a value greater than 500 supports the diagnosis of HCC without further imaging. A transthoracic echocardiogram showed a bilobed mass in the inferior portion of the right atrium:
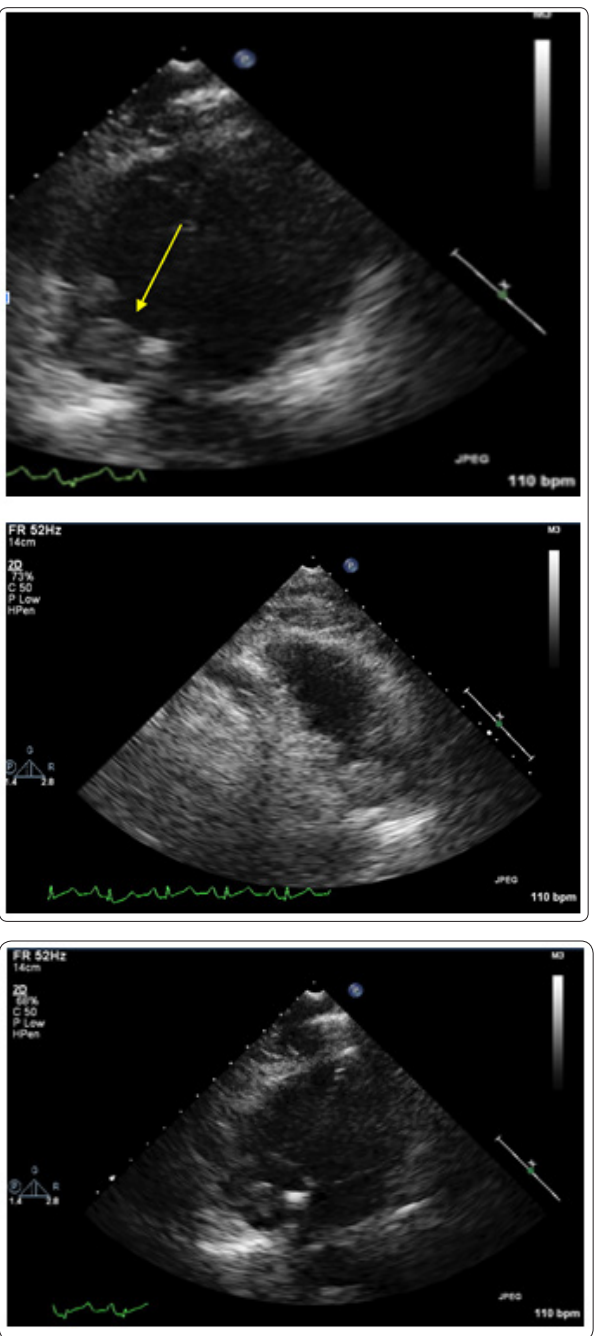

Magnetic resonance venography (MRV) which showed occlusion of right intrahepatic portal vein: 

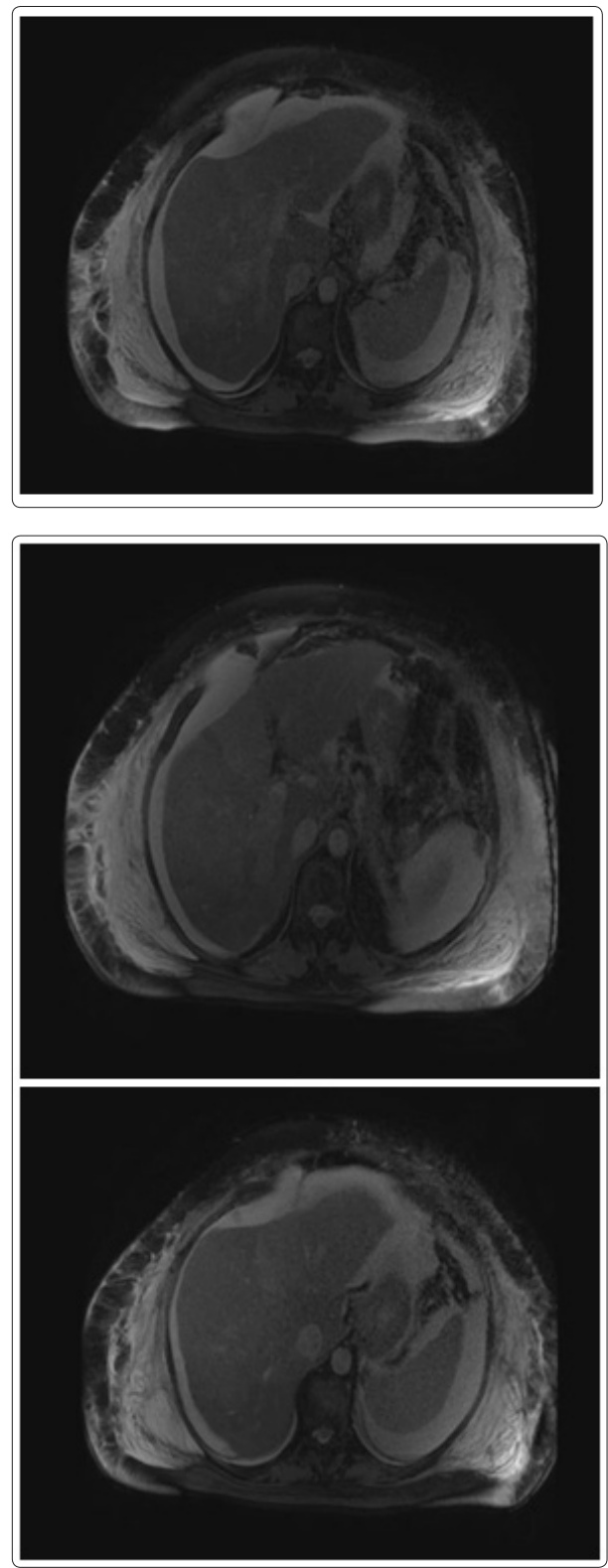

Fine needle aspiration biopsy with reticulin stain demonstrated a thickened hepatocellular plate consistent with trabecular hepatocellular carcinoma. According to the Barcelona-Clínic Liver Cancer (BCLC) staging system, the patient was classified as ChildPugh Class C. Based on the Eastern Cooperative Oncology Group (ECOG) Performance status, she had a score of 3 points and was thus staged end-stage HCC, with expected survival less than 3 months. No therapeutic interventions were offered by the time diagnosis was established as the patient's functional status had declined significantly and the disease was too advanced for possible improvement in life expectancy.

\section{Discussion}

The prevalence and incidence of HCC continues to rise annually. In the United States (US), the majority of HCC arises from viral hepatitis, with $16 \%$ cases attributed to $\mathrm{HBV}$ and $48 \%$ to $\mathrm{HCV}$ [1]. HBV-related HCC tends to develop 25-30 years after chronic infection [1]. Other risk factors include alcoholic liver disease, nonalcoholic steatohepatitis, and intake of aflatoxin-contaminated food, diabetes, and obesity [9]. This case illustrates the subtle presentation of HCC and that early diagnosis is key for treatment. The patient did not have any history of liver disease and denied use of alcohol, thus the cause of her cirrhosis was unknown and deemed cryptogenic. Testing for viral, autoimmune, and genetic conditions were negative. The patient's condition had deteriorated significantly throughout the hospital likely due intravascular volume depletion and decreased venous return from tumor metastasis causing progressive renal and liver failure. Given current guidelines based on HCC staging, the patient was classified as Child-Pugh Class B given vascular invasion. The guidelines recommend Sorafenib as the first line treatment option [5]. Although the current guidelines recommend against it, isolated case studies show improvement in survival in advanced HCC with IVC tumor/ thrombus who have adequate functional status and liver function [6]. We will discuss diagnostic strategy and alternative management of HCC with venovascular occlusion in the setting of renal failure.

Patients with HCC with metastasis to the right atrium present with clinical symptoms of right heart failure, including peripheral lower extremity edema, jugular venous distention, and ascites. Furthermore, sudden death has been reported due to ball valve thrombus syndrome [7]. The most common symptoms of cardiac metastasis reported are asymptomatic in 39.5\%, bilateral lower leg edema in $37.5 \%$ and exertional dyspnea in $31.3 \%$ [1]. To prevent sudden death due to this syndrome or pulmonary embolism, right atrial tumor resection is necessary [7]. Our patient presented with bilateral $3+$ pitting edema in her bilateral lower extremities, a sign of right-sided heart failure.

Malignant cardiac tumors, primary and secondary, occur more frequently in the right heart. Metastatic cardiac tumors develop 6-40 times more frequently than primary tumors and usually occur in the myocardium, followed by the epicardium, pericardium, and endocardium [7].

Myxoma is the most common benign tumor (50-70\%); angiosarcoma is the most common malignant tumor (30\%), followed by rhabdomyosarcoma (20\%) [8]. Most metastatic cardiac tumors originate from lung cancers, lymphomas, or breast cancers, but those resulting from gastrointestinal cancer or HCC are rare [7]. Our patient had an extremely rare case of both isolated right atrium metastasis of $\mathrm{HCC}$ as well as a cardiac tumor in the right atrium.

HCC metastases usually spread through intrahepatic blood vessels, lymphatics, or direct infiltration. Hematogenous extension may be the result of spread through the hepatic veins, portal veins, or inferior vena cava and this appears to have been the route of metastasis for our patient [5]. The mechanism of cardiac involvement is related to the tendency of this tumor to invade the vena cava, thus easily reaching the cardiac cavities [1].

Current surgical and medical treatment options are transcatheter arterial embolization (TAE), transcatheter arterial infusion chemotherapy (TAI) and radiotherapy [3]. Overall, all of the patients have an extremely poor prognosis regardless of the treatment strategy. The 5-year survival rates following surgery are $26 \%$ for cardiac metastases [8]. Thalidomide is hypothesized to be a useful angiogenesis inhibitor for IVC and RA metastases and new molecular target drugs, such as Sorafenib, are also being investigated [3]. Further studies need to investigate the current and novel treatment 
options currently available for both HCC and cardiac metastasis.

This case illustrates a rare cause of renal failure in a patient with no known liver disease, who was found to have advanced hepatocellular carcinoma with metastasis to the IVC and RA. This case reveals the importance of practitioners having a wide differential diagnosis. We recommend that echocardiography should be routinely performed for all patients with advanced HCC in order to detect cardiac metastasis early. Currently the prognosis for this disease is poor so further investigation into treatments is warranted.

\section{References}

1. Albackr HB (2014) A large Right atrial mass in a patient with hepatocellular carcinoma: Case report and literature review. Journal of the Saudi Heart Association 26: 174-178.

2. Harris PS, Hansen RM, Gray ME, Massoud OI, McGuire BM, et al. (2019) Hepatocellular carcinoma surveillance: An evidencebased approach. World J Gastroenterol 25: 1550-1559.

3. Kawakami M, Koda M, Mandai M, Keiko Hosho, Yoshikazu Murawaki, et al. (2013) Isolated metastases of hepatocellular carcinoma in the right atrium: Case report and review of the literature. Oncology Letters 5: 1505-1508.

4. Paraskevaidis IA, Michalakeas CA, Papadopoulos $\mathrm{CH}$, Anastasiou-Nana M (2011) Cardiac Tumors. ISRN Oncology 2011: 1-5.

5. Hartke J, Johnson M, Ghabril M (2017) The diagnosis and treatment of hepatocellular carcinoma. Semin Diagn Pathol 34: 153-159.

6. Gao H, Xu L, Zhang Y, Chen M (2014) Long-term survival of patients with hepatocellular carcinoma with inferior vena cava tumor thrombus treated with sorafenib combined with transarterial chemoembolization: Report of two cases and literature review. Chin J Cancer 33: 259-264.

7. Takaya H, Kawaratani H, Seki K (2017) A Patient with Hepatocellular Carcinoma with Isolated Right Atrial Metastases. Internal Medicine 56: 2589-2594.

8. Hoffmeier A, Sindermann JR, Scheld HH, Martens S (2014) Cardiac Tumors-Diagnosis and Surgical Treatment. Dtsch Arztebl Int 111: 205-211.
Copyright: (02020 Yaser Khalid, et al. This is an open-access article distributed under the terms of the Creative Commons Attribution License, which permits unrestricted use, distribution, and reproduction in any medium, provided the original author and source are credited. 